\title{
Carotenoid synthesis and function in plants: Insights from mutant studies in Arabidopsis*
}

\author{
Dean DellaPenna \\ Department of Biochemistry/200, University of Nevada, Reno, Nevada 89557, USA
}

\begin{abstract}
From a molecular and genetic perspective, the decade of the 1990s was truly unparalleled in the study of carotenoids. A combination of new technologies and approaches allowed the isolation of bacterial carotenoid biosynthetic genes and the subsequent isolation of higher plant homologs based on colour complementation in Escherichia coli. These genes provided a basis for molecular and transgenic studies of the carotenoid pathway in various eukaryotic organisms which, although not detailed in this article, critically and synergistically impacted the work described. For in-depth information of plant carotenoid molecular biology, readers are directed to a recent comprehensive review [F.X. Cunningham, E. Gantt. Annu. Rev. Plant Physiol. Plant Mol. Biol. 49, 557-583 (1998)]. The primary focus on this article is to review the identification and characterization of novel mutations in Arabidopsis, defining genes essential for xanthophyll synthesis in photosynthetic plant tissues. These mutations, both singly and in combination, allow dramatic alterations in the carotenoid composition of Arabidopsis photosystems in vivo, in the most extreme cases eliminating the synthesis of all wild-type xanthophylls (lutein, violaxanthin and neoxanthin). This work demonstrates a surprising plasticity in the carotenoid compositions that plant photosystems will accept in vivo and provides an insight into the functional role(s) of individual carotenoids in the photosystems.
\end{abstract}

\section{INTRODUCTION}

Carotenoids are integral components of higher plant photosystems, and their composition in photosynthetic plant tissues (lutein, $\beta$-carotene, violaxanthin and neoxanthin, in order of abundance) has been remarkably conserved throughout evolution. Carotenoids are multifunctional compounds serving as structural components of light-harvesting complexes (LHCs), accessory pigments for light harvesting, substrates for abscisic acid synthesis, components of photoprotection and scavengers of singlet oxygen. The biosynthesis of carotenoids in plants has been reviewed extensively in recent years and is only briefly described here.

The committed step to carotenoid synthesis is the formation of the first $\mathrm{C}_{40}$ compound phytoene by the head-to-head condensation of two molecules of GGDP (geranylgeranyl diphosphate) by phytoene synthase. Phytoene is subjected to a series of four sequential desaturation reactions, by two separate enzymes, to yield lycopene, which has eleven conjugated double bonds. Lycopene is then cyclized to $\beta$ carotene by two $\beta$-cyclizations or to $\alpha$-carotene by one $\beta$ - and one $\epsilon$-cyclization (Fig. 1 ). The two types of rings $(\epsilon$ and $\beta$ ) are produced by distinct enzymatic mechanisms [1] now known to be carried out by two structurally related enzymes, the lycopene $\beta$-cyclase and the lycopene $\epsilon$-cyclase [2]. The formation of $\epsilon$ rings and the production of $\beta, \epsilon$-carotenoids ( $\alpha$-carotene derivatives) are two of the key differences distinguishing carotenoid biosynthesis in plants from that in cyanobacteria, fungi and bacteria. $\alpha$ Carotene serves as the precursor for hydroxylation (twice) to lutein, the most abundant carotenoid in green plant tissues. $\beta$-Carotene is subjected to a series of hydroxylation and epoxidation reactions to yield

\footnotetext{
* Lecture presented at the 12th International Symposium on Carotenoids, Cairns, Australia, 18-23 July 1999, pp. 2205-2302.

Correspondence: E-mail: della_d@med.unr.edu
} 
the other common leaf xanthophylls: zeaxanthin, antheraxanthin, violaxanthin and neoxanthin (Fig. 1). The reversible epoxidation/de-epoxidation of zeaxanthin into violaxanthin (the xanthophyll cycle) is a light-dependent reaction thought to play an important role in adaptation to high light stress.

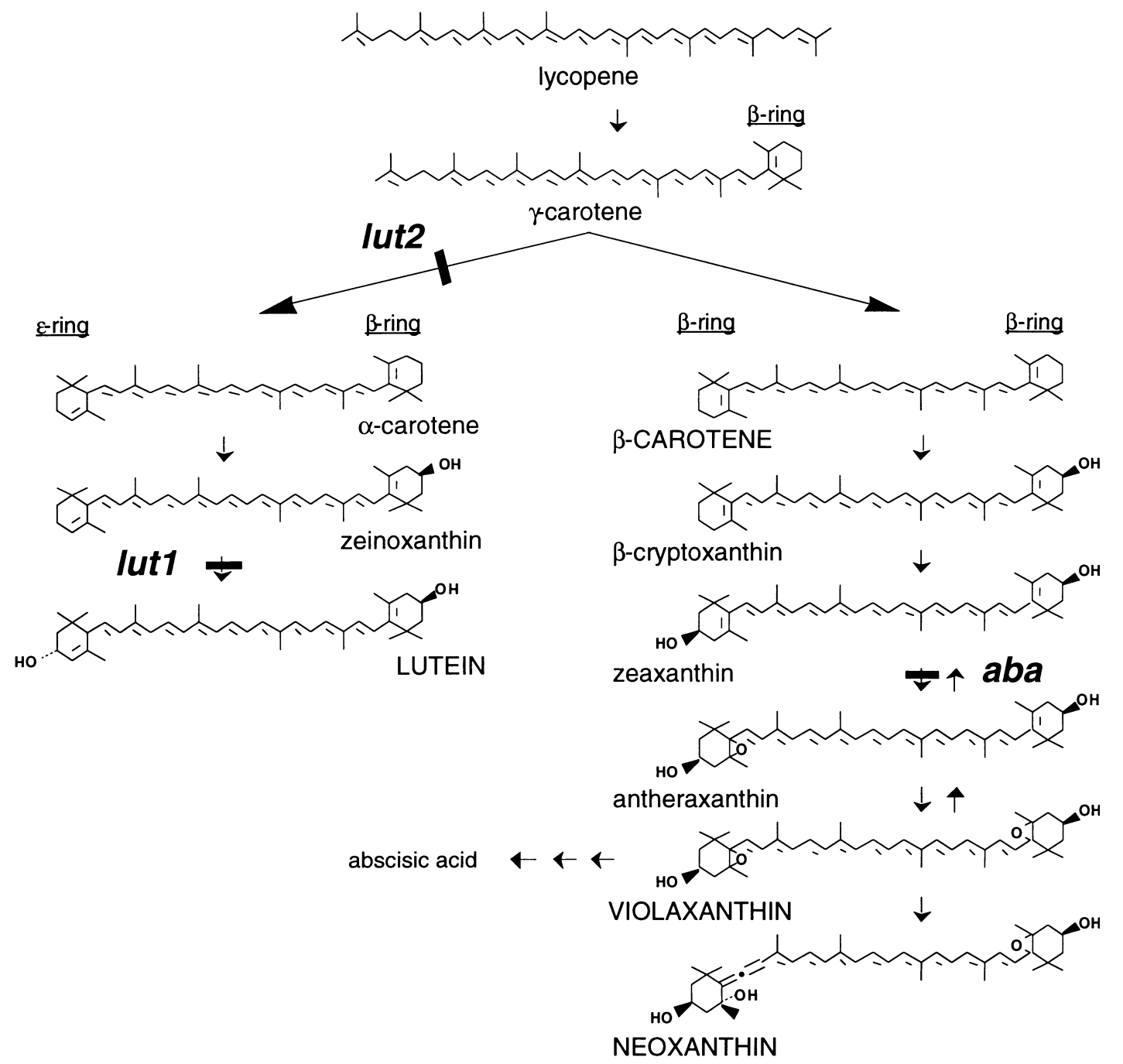

Fig. 1 Carotenoid biosynthetic pathways in plants. For clarity, only the portion of the biosynthetic pathway starting with lycopene is shown. The enzymatic steps blocked by the lut1, lut 2 and aba mutations are identified. Carotenoids that typically accumulate in photosynthetic tissues are given in capital letters.

While the carotenoid pathway has been known for several decades, the fact that most enzymes of the pathway are membrane-associated proteins that rapidly lose activity during isolation has hindered attempts at enzyme purification and study. The breakthrough in our understanding of the pathway and enzymes in recent years can be attributed largely to molecular and genetic approaches, which have together allowed the majority of plant carotenoid biosynthetic enzymes to be cloned (reviewed in [3]). These accomplishments have provided a wealth of information about the encoded proteins and the tools necessary to begin addressing many fundamental biochemical and molecular questions of the pathway. To this end, several studies have demonstrated that messenger RNA (mRNA) levels for early steps of the pathway are strongly upregulated in tissues such as flowers and fruits and to a much lesser extent during leaf development [4-6]. The availability of complementary DNAs (cDNAs) has also greatly aided biochemical studies which can now utilize large quantities of highly purified protein overexpressed in heterologous systems, rather than relying on partially purified, low specific activity natural sources $[7,8]$. 
While this allows individual enzyme activities to be studied in some detail, it provides little information about integration and regulation of the pathway in vivo and the function of individual carotenoids in the photosystems.

\section{Rationale for isolating carotenoid biosynthetic mutants in Arabidopsis}

Biochemical and biophysical approaches have provided significant advances in our understanding of the structural and functional roles of carotenoids in plant photosystems. However, these approaches are most often in vitro based and in vivo testing of the hypotheses developed has been limited. A complementary approach for such studies is molecular genetics, which allows one to genetically disrupt key components of pathways in vivo and assess the consequences on the pathway(s) or process(es) under study. The use of molecular genetic approaches has been a critical dimension in the study of carotenoid synthesis and function in bacterial systems, and provides an attractive means for critically testing the in vivo validity of current theories. The identification, characterization and utilization of mutant plant lines, in which the carotenoid composition of photosystems can be predictably manipulated at the genetic level, should allow analogous insights into the in vivo role of carotenoids in plant photosystem structure, assembly and function.

The studies described in this article represent ongoing research from several laboratories taking a molecular genetic approach to study the biosynthesis and function of carotenoids in the model plant Arabidopsis thaliana. To date, this work has helped define the enzymes responsible for the suite of native carotenoids produced in plants [2,9], critically tested the role of individual enzymes and xanthophylls in photosynthetic processes $[10,11]$ and provided insight into the integration of carotenoid synthesis with other components of the photosynthetic apparatus, namely the PQ (plastoquinone) pool and electron transport chain [12]. Through work in the author's laboratory various single and double mutant lines have been generated that are blocked at one or more steps in the carotenoid biosynthetic pathway and, as a result, show dramatically altered carotenoid profiles relative to wild-type Arabidopsis (Fig. 2). These carotenoid biosynthetic mutants are currently being used to investigate the physiological, structural and biochemical effects of altered carotenoid compositions on photosynthesis and photosystem structure and function in vivo. Because our understanding of the role of carotenoids in plants has been derived primarily from in vitro biochemical and biophysical studies, it is anticipated that these Arabidopsis mutants will be useful for the testing of current theories regarding the structural and functional role of specific carotenoids in plant photosystems.

\section{XANTHOPHYLL BIOSYNTHETIC MUTANTS OF ARABIDOPSIS}

Prior to 1995, only one locus affecting xanthophyll biosynthesis in the photosynthetic tissues of Arabidopsis had been identified, the $A B A l$ locus $(A B A=A B$ scisic $A$ cid deficient), the mutation of which disrupts zeaxanthin de-epoxidase, one of two xanthophyll cycle enzymes $[13,14]$. As a step towards advancing the understanding of xanthophyll biosynthesis, incorporation and function in plants, the author's laboratory has screened for and identified mutations defining two additional loci required for xanthophyll biosynthesis in Arabidopsis, LUT1 and LUT2 (LUT=LUTein deficient). Mutations at either locus result in defects in the synthesis of lutein, the most abundant xanthophyll in green plant tissues. Singly and in combination with the aba mutation, the lut mutations have allowed the genetic construction of five distinct mutant lines, which differ dramatically in their carotenoid composition relative to wildtype Arabidopsis (Fig. 3). In the remainder of this article, I will discuss the various single mutants, and end with a discussion from recent experiments in which we have introduced the $a b a$ mutation into each lut mutant background and characterized the effect of the combined mutations on growth, pigment profiles and nonphotochemical quenching (NPQ).

\section{The Arabidopsis aba1 mutation}

The abal mutation was discovered more than 15 years ago in a mutant screen designed to identify Arabidopsis mutants whose seed was desiccation intolerant and could be germinated in the absence of gibberellic acid synthesis [14]. The abal mutation is now known to identify the structural gene encoding the carotenoid biosynthetic enzyme zeaxanthin epoxidase, whose mutation virtually eliminates the 


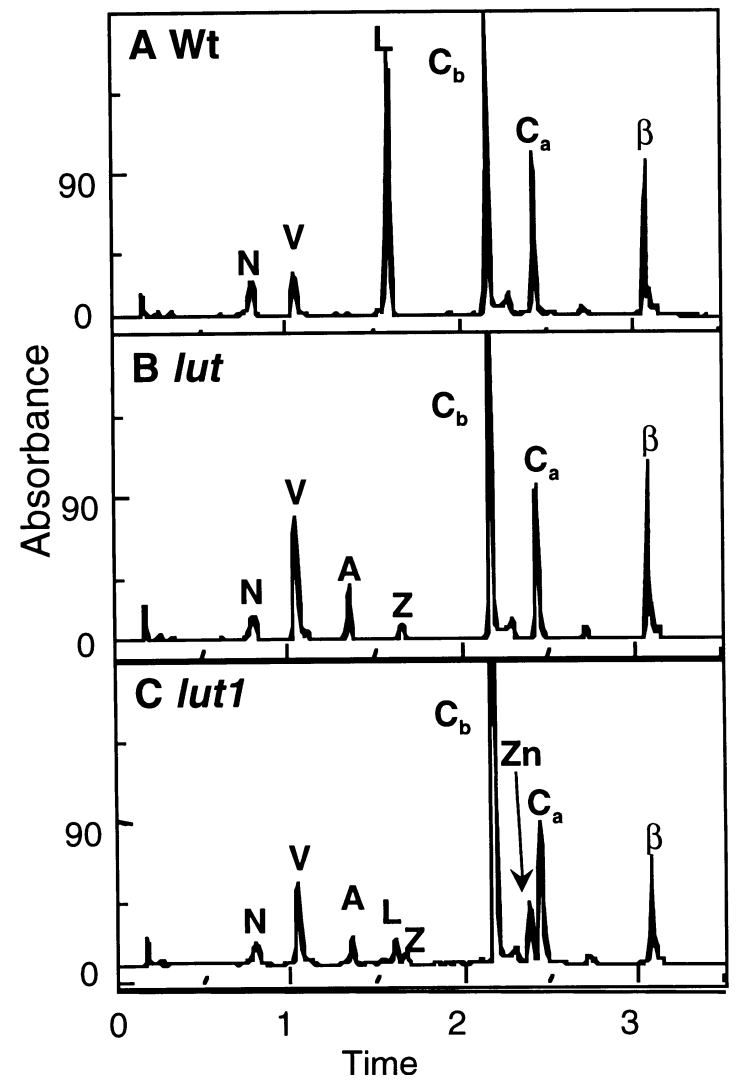

Fig. 2 Pigment analysis of wild-type, lut 2 and lut1 leaves. All panels are $\mathrm{C}_{18}$ high performance liquid chromatography (HPLC) separation of pigments from leaves of 4-week-old plants. (A) Wild-type; (B) lut2; (C) lut1. N, neoxanthin; V, violaxanthin; A, antheraxanthin; L, lutein; Zx, zeaxanthin; $\mathrm{C} b$, Chl $b$; $\mathrm{C} a$, Chl $a$; Zn, zeinoxanthin; $\beta, \beta$-carotene; wt, wild-type. Each profile represents absorbance at $440 \mathrm{~nm}$ of pigments extracted from $5 \mathrm{mg}$ fresh weight of tissue.

production of antheraxanthin, violaxanthin and neoxanthin in mutant tissues and results in their equimolar replacement with zeaxanthin [13]. The abal mutant has recently been used to study the effect of replacing violaxanthin and neoxanthin with zeaxanthin on LHC structure, chlorophyll fluorescence, photosynthesis and photoinhibition in vivo [15-17]. These studies have reported no significant differences in abal with respect to photosynthetic performance or amplitude of NPQ relative to wild-type, although NPQ developed faster and reverted slower in the dark in the mutant. LHC protein levels were also unaffected in the mutant, although a decrease in trimeric LHC in favor of monomers was observed, consistent with the decreased stability of the zeaxanthin containing abal LHC complexes [16,17].

\section{Isolation/characterization of Arabidopsis mutants defective in lutein synthesis}

To further our understanding of xanthophyll biosynthesis and function in higher plants, the author's laboratory has taken a molecular genetic approach to isolate and characterize novel mutations in Arabidopsis thaliana that result in defects in specific aspects of xanthophyll synthesis [9]. More than 4500 individual soil-grown EMS (ethylmethane sulfonic acid)-generated M2 mutant lines were screened individually for abnormal pigment profiles by high performance liquid chromatography (HPLC). The vast majority showed a wild-type pigment profile, but several lines impaired in their ability to accumulate xanthophylls were also identified (Fig. 2). These mutants could be divided into two groups: one group accumulated zeaxanthin, lacked violaxanthin and neoxanthin and was allelic with the previously identified abal locus, while a second group was impaired in lutein accumulation. This latter group, named lut for lutein deficient, was further divided into two classes: the lut 2 class had no detectable lutein 

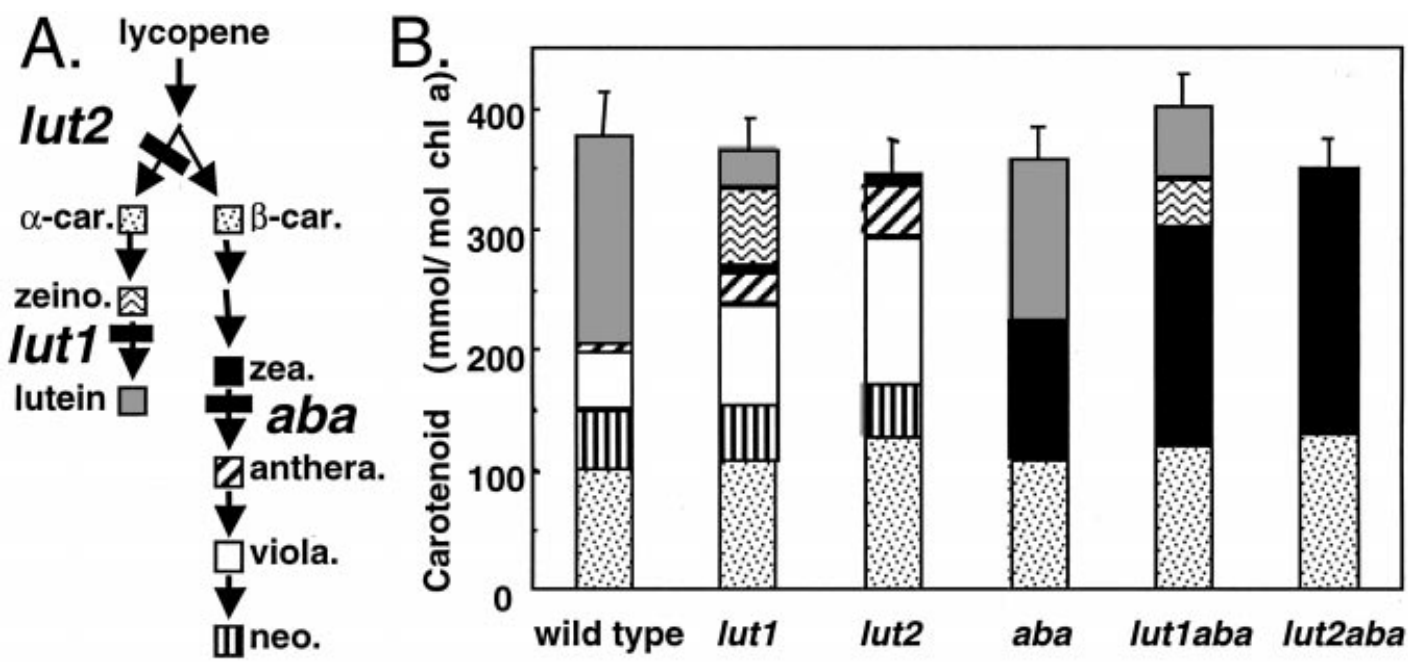

Fig. 3 Carotenoid pathway, mutant locations and pigment profiles. Panel A shows the carotenoid biosynthetic pathway in higher plant chloroplasts commencing with lycopene and the location of the lutl, lut 2 and abal mutations. Panel B shows the carotenoid content of mature green leaves in 21-day wild-type and the five indicated xanthophyll mutant lines. Each section of the bars corresponds to a specific carotenoid of the pathway (refer to coded boxes of Panel A). The standard deviations of the total pool of carotenoids per mole Chl $a$ are shown on each bar.

and increased amounts of $\beta$-carotene and xanthophyll cycle pigments; the lutl class also had severely reduced lutein, increased amounts of xanthophyll cycle pigments and accumulated a novel carotenoid not present in wild-type. These two lut loci represent the first mutations in higher plants specifically disrupting the synthesis of the $\alpha$-carotene derived xanthophyll lutein and have genetically defined the $\alpha$ carotene branch of the carotenoid pathway in plants [9].

\section{lut1 mutations disrupt $\epsilon$-ring hydroxylation}

All lut 1 alleles are recessive mutations that accumulate an additional carotenoid with an HPLC retention time, UV/visible and mass spectra characteristic of the monohydroxy $\beta, \epsilon$-carotenoid zeinoxanthin ( $\beta, \epsilon$-caroten-3-ol). The only difference between lutein and zeinoxanthin is the presence of a hydroxyl group on carbon 3 of the $\epsilon$-ring in lutein. The decrease in lutein and its partial replacement by its immediate precursor, zeinoxanthin, defines lutl as a mutation disrupting $\epsilon$-ring hydroxylation (Fig. 1). lut1 mutations are specific for $\epsilon$-ring hydroxylation, and do not impede other carotenoid biosynthetic reactions including, most significantly, $\beta$-ring hydroxylation. Thus, lutl genetically defines a minimum of two hydroxylation enzymes in the pathway, one specific for $\epsilon$-rings and a second, unaffected by the lut 1 mutation, specific for $\beta$-rings.

\section{lut2 mutations disrupt $\epsilon$-ring cyclization}

In contrast to lut l, lut 2 mutants do not accumulate any lutein precursors. The synthesis of $\beta, \beta$-carotenoids is unimpeded in lut 2 , and so lut 2 is not a $\beta$-cyclase or $\beta$-ring hydroxylase mutation. Based solely on biochemical data, lut 2 most likely affects $\epsilon$-cyclization, the committed step in the synthesis of lutein (Fig. 1). Recently, the lut 2 mutation has been complemented by transformation with a wild-type Arabidopsis $\epsilon$-cyclase cDNA driven by a constitutive promoter (data not shown). Thus, genetic biochemical and molecular complementation data support lut 2 being a disruption of the $\epsilon$-cyclase gene. The lut 2 mutations genetically define a minimum of two cyclization enzymes in the pathway, an $\epsilon$-cyclase and a $\beta$-cyclase. Interestingly, both lut 2 alleles are semidominant suggesting that $\epsilon$-cyclase is a ratelimiting enzymatic step for lutein synthesis and a key regulatory step in the production of $\alpha$ - and $\beta$-carotene derivatives $[2,9]$. 


\section{Carotenoid composition and function are altered in lut1 and lut2}

The loss of lutein (80-100\%) in lut1 and lut2 is compensated for by increases in the abundance of other carotenoids, most notably specific $\beta, \beta$-carotenoids (Fig. 3). lut 1 mutants accumulate the biosynthetic intermediate, zeinoxanthin, to approximately $50 \%$ of the wild-type lutein level and have elevated levels of the xanthophyll cycle carotenoids violaxanthin, antheraxanthin and zeaxanthin. lut 2 mutants have an even larger increase in xanthophyll cycle pigments, plus a smaller increase in $\beta$-carotene. Antheraxanthin and zeaxanthin normally accumulate transiently in response to high light stress due to de-epoxidation of violaxanthin, and in darkness are expoxidated back into violaxanthin. However, their accumulation in lut 1 and lut 2 is clearly not stress related as all plants were grown at a light intensity that did not induce the xanthophyll cycle in wild-type and their presence was not dark revertible in either mutant. Apparently, the bulk of these xanthophyll cycle pigments in the lut mutants are no longer in a location accessible to the epoxidase, most likely as a result of their preferential incorporation into LHC sites normally occupied by lutein.

Lutein is the most abundant carotenoid in all photosynthetic plant tissues and its synthesis and presence are evolutionarily conserved both in land plants and green algae. The apparent localization of lutein in the atomic structure of LHCII [18] and its requirement for optimal in vitro assembly of LHCs [19-21] has led to the assumption that lutein is critical for higher plant photosystem assembly and function. Yet, paradoxically, the complete elimination of lutein in a higher plant via the Arabidopsis lut mutants has no obvious deleterious effect on growth and development. The most reasonable explanation for the viability of the lut 1 and lut 2 plants is that some, or all, of the carotenoids that accumulate in its absence can functionally complement lutein, at least to some degree. In vitro studies support this hypothesis as, while less than optimal, various xanthophyll combinations enable LHC assembly in the absence of lutein.

The individual lutl, lut 2 and abal mutants have provided insight into the regulation of carotenoid biosynthesis and have demonstrated a surprising in vivo flexibility of plant LHCs with respect to carotenoids. In all three mutant lines, the total quantity of carotenoids did not change, but instead deletions were replaced by compensating molar increases in specific carotenoids (Fig. 3B). This is significant as it suggests that there is no net alteration in carbon flow to the pathway in mutations disrupting either branch of xanthophyll synthesis.

\section{Testing xanthophyll 'plasticity' with xanthophyll double mutants}

During the course of studies with single xanthophyll mutants, we began to consider the limitations of xanthophyll substitutions which would still enable viable assembly, light harvesting and photoprotection of higher plant photosystems in vivo. In all three single mutants, there was at least one 'native' xanthophyll still present: lutein in abal and neoxanthin and violaxanthin in lutl and lut 2 . In order to further test the minimum xanthophyll requirement of plant LHCs in vivo, we attempted to generate lut l/ $a b a 1$ and lut2/abal double mutant lines, the net effect of which would be to eliminate the synthesis of all 'native' leaf xanthophylls in both double mutant lines. Based on in vitro LHC assembly studies with various xanthophylls, one would predict detrimental effects on photosystem assembly/function in these double mutants. However, both double mutant lines were viable and photoauxotrophic at moderate light levels. The lutl/abal double mutant accumulates $\beta$-carotene, zeinoxanthin and zeaxanthin and is indistinguishable in growth from wild-type and either single parental mutant line. The lut $2 / a b a l$ double mutant only accumulates $\beta$-carotene and zeaxanthin, is approximately $50 \%$ smaller than wild-type or either single parental mutant, but is still viable. lut $2 / a b a l$ is also unique among the various mutant lines as it exhibits some seedling lethality in soil, which appears to be a function of both delayed greening and germination light intensity [11].

\section{Analysis of NPO in lut and aba single and double mutants}

We have also used the five different mutant lines shown in Fig. 3 to investigate the effects of altered xanthophyll compositions on the magnitude and timing of NPQ of chlorophyll fluorescence during exposure to high light [11]. In wild-type plants, the NPQ response was rapid, reaching a plateau after $80 \mathrm{~s}$ of illumination with high light (Fig. 4). However, in the lut mutants, which are defective in lutein 


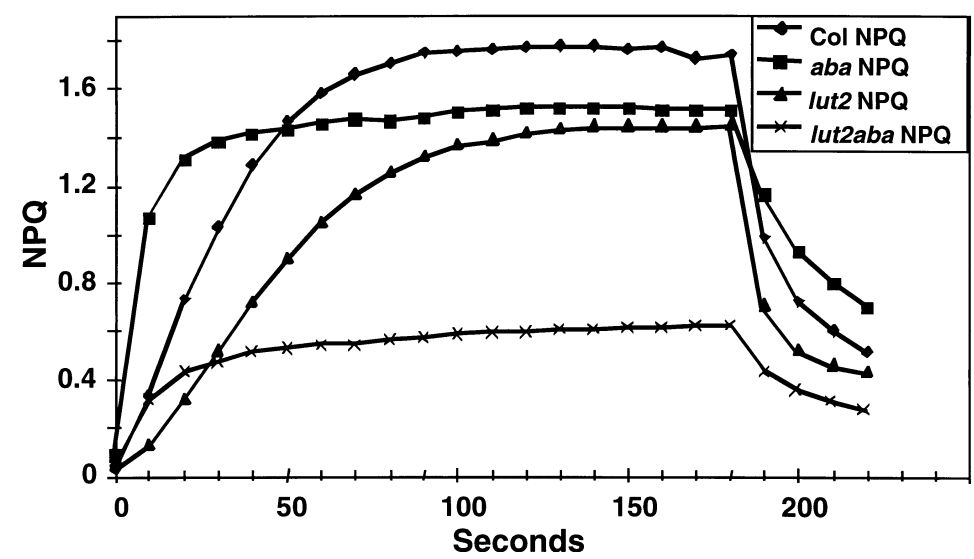

Fig. 4 Induction of nonphotochemical quenching (NPQ) in wild-type and xanthophyll mutant lines in lut2, abal and lut2/abal backgrounds. Plants were grown at $140 \mu \mathrm{mol} / \mathrm{m}^{2} / \mathrm{s}$ for $12 \mathrm{~h}$ cycles and dark adapted overnight. Fluorescence was measured before (zero time), during (0-180 s) and after (180-210 s, weak far red background light only) exposure to actinic light (photosynthetically active radiation PAR, of $1083 \mu \mathrm{mol} / \mathrm{m}^{2} / \mathrm{s}$ ). Three replicate plants were analyzed for each line and averaged.

synthesis, the induction of NPQ was significantly inhibited. After $10 \mathrm{~s}$ of illumination, the level of NPQ in lut 2 was only one-third that of wild-type, and $120 \mathrm{~s}$ were required before NPO plateaued, albeit at a significantly lower maximum level than wild-type. The patterns of NPQ induction and maximal NPQ levels were similar for lut1 and lut2 (only lut2 is shown in Fig. 4). In the lut2/abal double mutant, high constitutive levels of zeaxanthin restored the rapid phase of NPQ that was defective in the single lut 2 mutant. However, the maximal level of NPQ in the double mutant was much lower than wild-type or either of the single mutants.

The widely held view of the xanthophyll cycle is that zeaxanthin (and possibly antheraxanthin) are the only carotenoid(s) that contribute to NPQ [22-25]. The fact that the Arabidopsis lutein-deficient mutants have both delayed and reduced levels of NPQ, but still retain the enzymatic capacity to perform the xanthophyll cycle, suggests that lutein may also be involved directly or indirectly in NPQ. Although these results are far from conclusive, they suggest that the hypothesis that zeaxanthin and antheraxanthin are the only xanthophylls contributing to NPQ in plants needs re-examination.

While it is clear that the genetic removal of lutein from photosystems in vivo affects the rapid phase of NPQ, the question remains open as to whether lutein directly or indirectly impacts NPQ. A valid argument for an indirect contribution would be that the absence of lutein alters LHC antenna structure in such a way as to impede quenching. The alternative hypothesis is that lutein directly contributes to fluorescence quenching. This would necessitate that lutein has both the appropriate energy state and location to quench singlet chlorophyll. Both requirements can be met theoretically as the predicted excited $S_{1}$ energy state of lutein has a spectral overlap with the excited states of chlorophyll which would enable it to act as a quencher [26] and the proposed location of lutein in the atomic structure of the LHCII would be spatially appropriate [18]. While much work is required to define whether lutein directly or indirectly contributes to quenching, the genetic and photophysical results to date are consistent with the idea that lutein in some way acts as an integral component of NPQ.

\section{CONCLUSIONS AND PROSPECTS}

The studies described in this article have provided novel insights into carotenoid synthesis and function and demonstrated a surprising plasticity of plant photosystems in their xanthophyll composition. From in vitro and in vivo studies of photosystem assembly and function, and evolutionary and energetic considerations of the highly conserved carotenoid composition in photosynthetic plant tissues, more detrimental effects would be anticipated when entire classes and, in some cases, all wild-type xanthophylls are eliminated in Arabidopsis. Nonetheless, most xanthophyll deficient mutant lines are not 
visibly affected at moderate light conditions, indicating that various carotenoid combinations are capable of allowing the assembly of functional pigment-protein complexes under these conditions, although some combinations (e.g. lut2/abal) are clearly less efficient at assembly than others. As such, our results present a seeming paradox: Why would the 'native' carotenoid composition of higher plants be so highly conserved evolutionarily (lutein, $\beta$-carotene, violaxanthin, neoxanthin) when our mutants make it clear that plant photosystems are tremendously flexible in the carotenoids they will tolerate? One possible explanation to be tested in the near future is that the 'native' carotenoid composition represents, as a group, those carotenoids best suited to provide the full range of structural and functional flexibility required for photosynthesis in a natural environment. Answering these and other questions posed by mutations affecting carotenoid synthesis in Arabidopsis will be the focus of future studies in my laboratory.

\section{REFERENCES}

1 G. Britton. In Plant Pigments (T. Goodwin, ed.), pp. 133-182. Academic Press, San Diego, CA (1988).

2 F. X. Cunningham Jr, B. J. Pogson, Z. Sun, K.. A. McDonald, D. DellaPenna, E. Gantt. Plant Cell 8, 1613-1626 (1996).

3 F. X. Cunningham, E. Gantt. Annu. Rev. Plant Physiol. Plant Mol. Biol 49, 557-583 (1998).

4 M. Kuntz, J. L. Evrard, A. d'Harlingue, J. H. Weil, B. Camara. Mol. Gen. Genet. 26, 156-163 (1989).

5 M. Kuntz, S. Romer, C. Suire, P. Hugueney, J. H. Weil, R. Schantz, B. Camara. Plant J 2, 25-34 (1992).

6 G. Giuliano, G. E. Bartley, P. A. Scolnik. Plant Cell 5, 379-387 (1993).

7 G. Sandmann. J. Plant Physiol. 43, 444-447 (1994).

8 N. Misawa, M. R. Truesdale, G. Sandmann, P. D. Fraser, C. Bird, W. Schuch, P. M. Bramley. J. Biochem. 116, 980-985 (1994).

9 B. J. Pogson, K. McDonald, M. Truong, G. Britton, D. DellaPenna. Plant Cell 8, 1627-1639 (1996).

10 K. K. Niyogi, O. Bjorkman, A. R. Grossman. Proc. Natl. Acad. Sci. USA 94, 14 162-14 167 (1997).

11 B. J. Pogson, K. K. Niyogi, O. Bjorkman, D. DellaPenna. Proc. Natl. Acad. Sci. USA 95, 13 324-13 329 (1998).

12 S. R. Norris, T. R. Barrette, D. DellaPenna. Plant Cell 7, 2139-2149 (1995).

13 C. D. Rock, J. A. D. Zeevaart. Proc. Natl. Acad. Sci. USA 88, 7496-7499 (1991).

14 M. Koornneef, M. L. Jorna, D. L. C. Brinkhorst van der Swan, C. M. Karssen. Theor. Appl. Genet. 61, 385-393 (1982).

15 C. D. Rock, N. R. Bowlby, S. Hoffmann-Benning, J. A. D. Zeevaart. Plant Physiol. 100, 1796-1801 (1992).

16 F. Tardy, M. Havaux. J. Phytochem. Phytobiol. 34, 87-94 (1996).

17 V. Hurry, J. M. Anderson, W. S. Chow, C. B. Osmond. Plant Physiol. 113, 639-648 (1997).

18 W. Kuhlbandt, D. N. Wang, Y. Fujiyoshi. Nature 367, 614-621 (1994).

19 F. G. Plumley, G. W. Schmidt. Proc. Natl. Acad. Sci. USA 84, 145-150 (1987).

20 H. Paulsen, U. Rumler, W. Rudiger. Planta 181, 204-211 (1990).

21 H. Paulsen, B. Finkenzeller, N. Kuhlein. Eur. J. Biochem. 215, 809-816 (1993).

22 E. Pfundel, W. Bilger. Photosynth. Res. 42, 89-109 (1994).

23 B. Demmig Adams, W. W. Adams III. Planta 198, 460-470 (1996).

24 B. Demmig Adams, W. W. Adams, III. Trends Plant Sci. 1, 21-26 (1996).

25 A. M. Gilmore. Physiol. Plant. 99, 197-209 (1997).

26 H. A. Frank, A. Cua, V. Chynwat, A. Young, A. D. Gosztola, M. R. Wasielewski. Photosynth. Res. 41, 389-395 (1994). 